ISSN 1028-821X. Radiofiz. Electron. 2019. Vol. 24, No. 3: 45-60

DOI: https://doi.org/10.15407/rej2019.03.045

УДК 621.385.633.

\title{
М.В. Мипьчо
}

Институт радиофизики и электроники им. А.Я. Усикова НАН Украины

12, ул. Акад. Проскуры, Харьков, 61085, Украина

E-mail: astis@ire.kharkov.ua

\section{Исспедование и проектирование отборника мощности дия эпектровакуумного умножитепя частоты субмилпиметрового диапазона волн}

\begin{abstract}
Предмет и цель работы. В настоящее время существует проблема создания удобных в эксплуатации генераторов для освоения коротковолновой части субмиллиметрового диапазона волн. В генераторах типа ЛОВ (лампа обратной волны) и клинотронах при укорочении длины волны возрастают трудности, связанные с уменьшением размеров замедляюших систем и повышением требований к качеству электронных пучков. Эти требования можно значительно ослабить, используя электровакуумные умножители частоты. В статье описано численное исследование работы отборника мощности в электронных умножителях частоты субмиллиметрового диапазона волн. Цель этих исследований - оценка достижимой выходной мощности в таких умножителях частоты.

Методы и методология работы. Для вычисления КПД отборника мощности проводится анализ трехмерного движения электронных сгустков в заданном электромагнитном поле бегущей волны в замедляющей системе типа «гребенка». Для численного анализа используется разработанная автором программа «Клинотрон». Для описания распределения в пространстве напряженности электрического поля используется строгий аналитический метод конформных отображений. При этом в аналитических выражениях учитываются сразу все пространственные гармоники поля и строго описывается сингулярность поля вблизи ребер гребенки.

Результаты работы. Проведено численное исследование влияния параметров отборника мощности на выходные параметры умножителя. Определены режим и конструктивные параметры отборника мощности умножителя частоты для диапазонов волн $\lambda=0,5$ мм и $\lambda=0,3$ мм.

Заключение. Проведенное исследование показало, что при существующих технологических возможностях реально создать умножители частоты для диапазонов волн $\lambda=0,5 \ldots 0,3$ мм с выходной мощностью порядка десятков милливатт. Необходима также оптимизация модулятора и группирователя электронных сгустков в умножителе частоты. Ил. 4. Табл. 4. Библиогр.: 20 назв.
\end{abstract}

Ключевые слова: электроника, клинотрон, группировка, умножитель частоты, численный счет.

В настоящее время для освоения коротковолновой части субмиллиметрового (субмм) диапазона волн практически нет простых и удобных в эксплуатации генераторов. Для этой части диапазона могут использоваться релятивистские генераторы, созданные на основе ускорителей, или генераторы типа МЦР (мазеры на циклотронном резонансе), использующие сверхпроводящие магнитные системы для создания очень больших магнитных полей. Для коротковолновой части субмм-диапазона также используется умножение сигнала от лампы об- ратной волны (ЛОВ) на полупроводниковом элементе (диоды с барьером Шоттки), однако выходная мощность таких устройств очень мала (микроватты).

В ИРЭ НАН Украины были разработаны генераторы типа ЛОВ - клинотроны [1]. Эти генераторы конструктивно просты и удобны в эксплуатации. Они перекрывают длинноволновую часть субмм-диапазона до 0,55 мм. Однако дальнейшее продвижение в коротковолновую часть диапазона затруднено. Главная трудность - создание замедляющих систем типа 


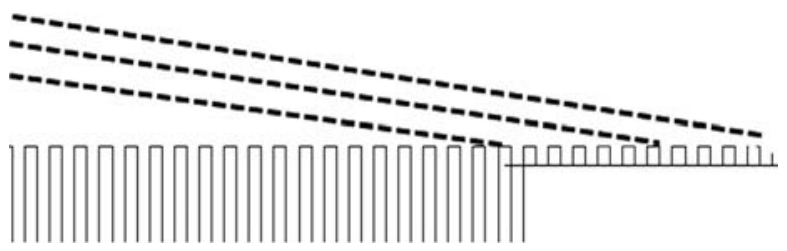

Puc. 1. Схема электровакуумного умножителя частоты. Показаны две последовательно расположенные гребенки и ленточный электронный пучок. Первая гребенка (слева) - модулятор, вторая (справа) - отборник мощности на кратной частоте

«гребенка» с очень узкими щелевыми резонаторами ( 0,01 мм).

Для ЛОВ этого диапазона могут использоваться мелкоструктурные замедляющие системы с напылением золота на алмазной подложке [2], поскольку алмаз является диэлектриком, обладая при этом хорошей теплопроводностью. К сожалению, такие технологии для нас недоступны.

Для ЛОВ субмм-диапазона в России разработана специальная технология изготовления мелкоструктурных периодических систем [3]. Из тонкой медной фольги методами фотолитографии вытравливаются элементы сложной формы, из которых можно составить период замедляющей системы сложной формы (например, типа цепочки связанных резонаторов). Эти элементы из медной фольги серебрятся и собираются на специальных приспособлениях в набор, образующий периодическую структуру со сложной геометрией. Этот набор пластин сжимается и прогревается до температуры плавления серебра. После такой пропайки образуется замедляющая система с периодом в сотые доли миллиметра. На основе такой технологии удалось разработать ЛОВ до длины волны 0,2..0,3 мм [4]. Выходная мощность этих приборов - единицы милливатт.

Существующие у нас технологические возможности не позволяют получить периодические структуры с таким малым периодом.

Для продвижения в коротковолновую часть субмм-диапазона могут оказаться перспективными умножители частоты (рис. 1).

В отборниках мощности умножителя можно использовать режим синхронизма электронов со скоростью высших пространственных гармоник больших номеров. При этом период замедляющей системы увеличивается в несколько раз. Следует пояснить, что такой режим не удается использовать в обычных генераторах типа ЛОВ, так как этому препятствует конкуренция типов колебаний. При одном и том же рабочем напряжении выполняются условия синхронизма и для желаемой гармоники поля, и для -1-й пространственной гармоники более длинноволнового типа колебаний. В таких условиях возбуждается длинноволновый тип колебаний, поскольку его поле слабее затухает при удалении от поверхности замедляющей системы и сильнее связано с электронным потоком.

В отборнике умножителя для возбуждения колебаний, кроме условия синхронизма электронов с полем гармоники, требуется также выполнить условие строгой кратности частоты модулятора умножителя частоте колебаний в отборнике мощности. Эти два условия можно выполнить одновременно только для одной пространственной гармоники, и поэтому конкуренции типов колебаний в умножителях нет.

Важное достоинство умножителей - отсутствие понятия пускового тока. В обычных генераторах, в режиме малых амплитуд поля, сгруппированная часть тока пропорциональна току пучка и амплитуде поля. Отдаваемая полю мощность пропорциональна произведению поля на сгруппированный ток, т. е. квадрату амплитуды поля и току пучка. Потери энергии в металле замедляющей системы также пропорциональны квадрату амплитуды поля. Превышение отдаваемой пучком мощности над потерями энергии обеспечивается только при достаточно большом токе пучка - пусковом токе. Это условие возбуждения колебаний в генераторе.

В умножителе сгруппированный ток обеспечивается модулятором и слабо зависит от поля в отборнике. Поэтому мощность, отдаваемая электронами полю, пропорциональна амплитуде поля, а не ее квадрату. При очень малых амплитудах поля квадрат амплитуды всегда будет много меньше амплитуды и поэтому всегда найдется настолько малое значение амплитуды, что пропорциональные квадрату амплитуды потери энергии окажутся меньше мощности, отдаваемой электронами полю. Это условие возбуждения и нарастания амплитуды колебаний в отборнике мощности. Поэтому даже при невозможности обеспечить высокое 
качество электронного пучка в умножителе, при выполнении резонансных условий и группировке электронов в достаточно компактные сгустки, будет получен выходной сигнал, хотя и малой мощности.

Известны экспериментальные работы по электровакуумным умножителям частоты в миллиметровом диапазоне волн (например, $[5,6])$. В качестве модулятора в этих работах использовался резонатор клистрона 3-см диапазона. На этот резонатор подавалась высокочастотная энергия от внешнего генератора мощностью около 50 Вт. Отборником мощности служил отрезок замедляющей системы типа гребенка в волноводе. Экспериментально исследовалось умножение в 8- и 2-мм диапазонах (на разных гребенках). При больших кратностях умножения был получен сигнал с мощностью в несколько милливатт.

В работах [7,8] была впервые показана возможность использовать электронный умножитель частоты в субмм-диапазоне волн. Экспериментальный прибор представлял собой двухсекционный клинотрон с двумя последовательно расположенными замедляющими системами типа «гребенка». Ленточный электронный пучок проходил последовательно над обеими замедляющими системами (рис. 1). Первая секция работала как клинотрон 3-мм диапазона, а вторая - как отборник мощности в диапазоне 0,9 мм. Было показано, что подобрав положение пучка и параметры гребенок, можно получить трехкратное умножение частоты с длиной волны 0,93 мм и выходной мощностью порядка единиц милливатт. При этом для входа в отборник использовалась только удаленная от поверхности первой гребенки часть пучка, где происходила модуляция электронов по скорости и плотности.

Наши попытки создать умножитель на таком же принципе, когда в одном электровакуумном приборе сочетались и генератор умножаемого сигнала, и модулятор электронного пучка, и отборник мощности, на диапазон $\lambda=0,5$ мм, оказались неудачными. Стало очевидным, что в субмм-диапазоне требуется оптимизировать как модулятор, так и отборник мощности. В «объединенном» варианте умножителя, где используется один и тот же электронный пучок, подобрать его оптимальное положение как для генератора-модулятора, так и для отборника мощности очень сложно. Поэтому мы планируем проверить также вариант с «запиткой» модулятора от внешнего генератора (типа ЛОВ или клинотрон).

Для успешного проектирования электронного умножителя частоты требуется раздельное исследование отборника мощности и группирователя электронных сгустков на основе надежных расчетных моделей. Целью таких исследований является оценка возможных выходных параметров этих устройств и поиск оптимального сочетания конструктивных параметров для их реализации.

В этой работе теоретически и численно исследуется только отборник мощности при сочетании параметров, характерных для субмм-диапазона волн и доступных для реализации.

1. Оценка области занимаемой полем рабочей гармоники. Можно показать, что область, занятая высокочастотным (ВЧ) полем гармоники над поверхностью периодической структуры (замедляющей системы), определяется только рабочим напряжением и частотой и не зависит от номера рабочей гармоники и параметров замедляющей системы. Как известно, любая пространственная гармоника представляет собой бегущую волну и описывается формулой:

$$
E(x, y)=E_{0} \exp [j(-\beta x+\omega t)] \exp \left(-\beta^{\prime} y\right),
$$

где $x$ - продольная координата, параллельная поверхности гребенки; $y$ - поперечная координата (расстояние от поверхности гребенки); $\beta$ продольная постоянная распространения; $\beta^{\prime}-$ поперечная постоянная распространения.

Параметры гармоники связаны соотношениями:

$\beta=\frac{2 \pi}{\Lambda}=\frac{\omega}{v_{0}}=\frac{\varphi_{n}}{S}$,

где $\Lambda$ - замедленная длина волны гармоники; $\omega$ - круговая частота; $v_{0}$ - фазовая скорость гармоники; $\varphi_{n}-$ сдвиг фазы гармоники не период гребенки; $S$ - период гребенки;

$\beta^{\prime}=\sqrt{\beta^{2}-k^{2}}=\beta \sqrt{1-\left(\frac{k}{\beta}\right)^{2}}=\beta \sqrt{1-\left(\frac{\Lambda}{\lambda}\right)^{2}}$,

где $k=2 \pi / \lambda(\lambda-$ длина волны в свободном пространстве). 
Если скорость электронов равна скорости пространственной гармоники (условие синхронизма), то замедление гармоники $\lambda / \Lambda$ связано с рабочим напряжением $U_{a}$ выражением:

$\frac{\lambda}{\Lambda}=\frac{505}{\sqrt{U_{a}}}$,

где $U_{a}$ - рабочее напряжение в вольтах.

Очевидно, что в нерелятивистских приборах $\beta^{\prime} \approx \beta$, так как при $U_{a}=2500 \mathrm{~B}(\Lambda / \lambda)^{2}=0,01, \mathrm{a}$ при $U_{a}=4000 \mathrm{~B}(\Lambda / \lambda)^{2} \approx 0,016$.

Убывание амплитуды поля гармоники при удалении на $y$ от поверхности гребенки описывается формулой:

$$
\begin{aligned}
& E(y)=E(0) \exp (-\beta y)= \\
& =E(0) \exp (-2 \pi y / \Lambda)= \\
& =E(0) \exp \left(-2 \pi \frac{505}{\sqrt{U_{a}}} \frac{y}{\lambda}\right) .
\end{aligned}
$$

Численная оценка для $\lambda=0,5$ мм и $\lambda=0,3$ мм при $U_{a}=4000$ В представлена в табл. 1.

Видно, что в субмм-диапазоне волн процесс взаимодействия поля с электронным пучком происходит только в очень узком слое у поверхности гребенки шириной всего 0,01...0,015 мм. Это обязательно следует учитывать при формировании и фокусировке электронных пучков в умножителях.

2. О параметрах замедляющей системы отборника мощности. Основным фактором, определяющим возможность реализации конкретной гребенки, является ширина щелевых резонаторов, образующих гребенку. Наиболее доступным для изготовления гребенок является электроискровой метод порезки щелей. Минимальная доступная толщина проволоки для нарезки щелей составляет $0,03 \ldots 0,04$ мм.

Таблица 1. Убывание амплитуды $\boldsymbol{E}$-поля рабочей гармоники при удалении от поверхности гребенки

\begin{tabular}{|c|c|c|}
\hline $\begin{array}{c}\text { Длина } \\
\text { волны } \lambda, \\
\text { в мм }\end{array}$ & $\begin{array}{c}\text { Расстояние } \\
\text { от поверхности } \\
\text { гребенки, } y, \text { в мм }\end{array}$ & $\begin{array}{c}\text { Убывание амплитуды } \\
\text { поля рабочей гармоники, } \\
\text { в разы }\end{array}$ \\
\hline 0,5 & 0,01 & 2,72 \\
& 0,02 & 7,39 \\
0,3 & 0,03 & 20,10 \\
& 0,01 & 5,31 \\
& 0,015 & 12,24 \\
\hline
\end{tabular}

С учетом разбивки щелей при нарезке минимальная ширина щелей оказывается равной $\sim 0,05$ мм. При этом минимальный период гребенки будет равен $0,1 \ldots 0,08$ мм.

Оценим минимальную длину волны генератора, которую можно получить при таком периоде гребенки. Из условия синхронизма (равенства скорости электронов и скорости рабочей пространственной гармоники) можно получить такое соотношение:

$$
\left|\frac{\varphi}{\pi}+2 n\right|=\frac{2 S}{\lambda} \frac{505}{\sqrt{U_{a}}},
$$

где $n$ - номер рабочей пространственной гармоники; $\varphi$ - фазовый сдвиг на период гребенки основной пространственной гармоники; $U_{a}-$ рабочее напряжение в вольтах.

Для разрабатываемых в ИРЭ НАН Украины клинотронов, работающих в субмм-диапазоне при оптимальных фазовых сдвигах $\varphi \approx 0,3 \pi$ и анодном напряжении не более $5000 \mathrm{~B}$, минимальная достижимая длина волны на $-1-$ й пространственной гармонике при периоде $S \approx$ $\approx 0,08$ мм составляет $\lambda \approx 0,68$ мм. Эти же оценки применимы и для отборника мощности электронных умножителей частоты, работающих на -1-й пространственной гармонике.

Поскольку умножители имеет смысл разрабатывать в той области длин волн, которая недоступна клинотронам, из приведенных выше оценок можно сделать вывод, что для отборника мощности следует использовать работу на высших пространственных гармониках с номером $|n|>1$. Для увеличения периода $S$ можно было бы использовать и +1-ю гармонику, однако для генератора следует выбирать отрицательные гармоники, когда основная волна движется навстречу потоку электронов и обеспечивает обратную связь в системе. Поэтому в отборнике мощности будем использовать -2-ю пространственную гармонику поля.

При разработке замедляющей системы для отборника мощности желательно, чтобы в гребенке была поверхностная волна (медленная основная гармоника). При этом расстояние от поверхности гребенки до экрана, если оно больше нескольких периодов гребенки, практически не влияет на дисперсионную характеристику гребенки. Это удобно при проектировании и изготовлении прибора. При работе на 
быстрой волне замедляющая система должна рассчитываться как объемный резонатор и требует точной установки экрана относительно поверхности гребенки.

Оценим, какие ограничения накладывает требование работы на поверхностной волне на параметры гребенки. В замедляющей системе существует режим скольжения волны, когда она переходит из поверхностной в объемную. Режим скольжения определяется условием $\beta^{\prime}=0$. Из (1) следует, что в этом режиме $\beta=k$. Поверхностную волну можно обеспечить при условии $\beta>k$ или

$$
\frac{\varphi}{\pi}>\frac{2 S}{\lambda} \text {. }
$$

Рассчитаем параметры гребенки для $\lambda=$ $=0,5$ мм, анодного напряжения $U_{a} \approx 4000 \mathrm{~B}$ для работы на -2-й пространственной гармонике поверхностной волны. Период гребенки рассчитывается по формуле (2) из условия синхронизма. Выбрав $\varphi=0,5 \pi$, получим период $S=0,11$ мм. По формуле (3) проверяем, что при $\varphi>0,44 \pi$ волна будет поверхностной (для этого мы выбрали достаточно большой фазовый сдвиг $\varphi$ ).

Ширину щели $l$ выбираем равной 0,08 мм. Такая широкая щель нужна для того, чтобы амплитуда -2-й гармоники была близкой к максимуму. Для амплитуд пространственных гармоник $E_{n}$ обычно используют приближенную формулу [9]

$\frac{E_{n}}{E_{0}}=\frac{l}{S} \frac{\sin \left(\beta_{n} l / 2\right)}{\beta_{n} l / 2}$,

где $E_{0}$ - среднее $E$-поле на «раскрыве» щели гребенки; $S$ - период гребенки; $l$ - ширина щелевого резонатора; $\beta_{n}=\varphi+2 n-$ постоянная распространения $n$-й пространственной гармоники; $\varphi$ - фазовый сдвиг на период для основной пространственной гармоники.

Анализ этой формулы показывает, что для -2-й пространственной гармоники при $\varphi=$ $=0,4 \ldots 0,6 \pi$ при широких щелях амплитуда этой гармоники будет расти при увеличении ширины щелей. Мы выбрали щель 0,08 мм, чтобы на ширину ламели осталось 0,03 мм.

Глубина щелей в гребенке рассчитывается достаточно точно по формулам, в которых строго учитывается распределение поля

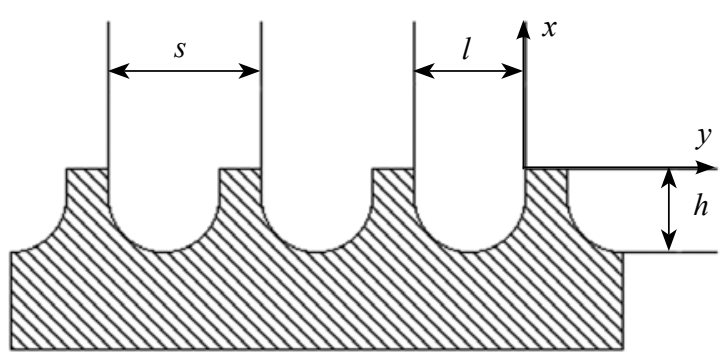

Puc. 2. Гребенка отборника мощности для работы на -2-й пространственной гармонике в диапазоне волн $\lambda=0,5$ мм $(s=0,11 \mathrm{мм} ; l=0,08 \mathrm{mм} ; h=0,06 \mathrm{мM})$

в замедляющей системе [10]. Для выбранных выше параметров гребенки глубина щелевых резонаторов составила $h=0,06$ мм. Проверка по дисперсионному уравнению работы [9] показала, что фазовый сдвиг на период $\varphi=0,5 \pi$ в такой гребенке будет при рабочем напряжении $U_{a}=4021$ В и длине волны $\lambda=0,5006$ мм (при работе на -2-й пространственной гармонике).

Следует отметить, что генераторы типа «клинотрон», в которых используется замедляющая система «гребенка в прямоугольном волноводе», работают на таких колебаниях замедляющей системы, при которых амплитуда поля вдоль ламелей гребенки изменяется очень слабо и в области на торцах гребенки поле практически не проникает. Такие колебания энергетически наиболее выгодны для взаимодействия с электронным потоком, и колебания с другим распределением поля с ними не конкурируют. Поэтому рабочие характеристики таких генераторов могут рассчитываться в предположении, что распределение поля в гребенке является двумерным. Это было установлено сравнением двумерных и трехмерных дисперсионных характеристик гребенок и подтверждено экспериментально $[10,11]$. Использование для расчета реального распределения поля мощных современных программ позволило подтвердить, что распределение поля над гребенкой действительно является практически двумерным [12]. В этой работе мы также предполагаем, что распределение поля не изменяется вдоль ламелей гребенки.

При изготовлении гребенки путем нарезки щелевых резонаторов электроискровым способом дно щелей всегда имеет скругленные углы (рис. 2).

Влияние «скругления» дна щелевых резонаторов на дисперсионные характеристики 
гребенки исследовалось теоретически и экспериментально в [13]. Показано, что при расчете гребенки со «скругленными» углами можно использовать формулы для гребенки с прямоугольной формой щелей, если считать глубиной щелевых резонаторов расстояние от поверхности гребенки до наиболее глубокого места на скругленном дне (рис. 2). Многочисленные проверки при изготовлении реальных генераторов подтвердили правильность этого вывода.

На рис. 2 показано, как выглядит рассчитанная выше гребенка. Хотя эта гребенка по виду сильно отличается от обычной, экспериментальная проверка показала, что ее дисперсионная характеристика хорошо совпадает с расчетной для гребенки с прямоугольной формы щелей.

Масштабным моделированием можно уменьшить все размеры этой гребенки с коэффициентом 0,6 и получить размеры гребенки для диапазона $\lambda=0,3$ мм. При этом рабочее напряжение и фазовый сдвиг на период останутся прежними. Округляя значения, получим такие размеры: $S=0,07$ мм; $l=0,05$ мм; $h=0,04$ мм. Проверка по дисперсионному уравнению [6] показала, что фазовый сдвиг $\varphi=0,5 \pi$ в такой гребенке будет при рабочем напряжении $U_{a}=3912$ В и длине волны $\lambda=0,323$ мм (при работе на -2-й пространственной гармонике). При этом волна будет поверхностной, поскольку режим скольжения в такой гребенке наступает при $\varphi=0,434 \pi$.

Важно отметить, что ширину щелей 0,05 мм еще можно получить на доступном нам оборудовании. Толщина ламелей 0,02 мм также достижима при уплотнении безкислородной меди предварительной ковкой - «нагартовкой».

Для дальнейшего укорочения длины волны нужно повышать рабочее напряжение или переходить на более высокий номер пространственной гармоники и работать не на поверхностной, а на быстрой объемной волне.

3. Методика расчета электронного кПД в отборнике мощности. Для анализа движения электронов в генераторах типа ЛОВ и клинотронах нами была разработан специальный пакет программ «Клинотрон», в котором учтены многие параметры, влияющие на энергообмен электронов с ВЧ-полем [7, 14, 15]. Эти же программы мы будем использовать для модели- рования работы отборника мощности в умножителе. Анализируется стационарный режим прибора, когда амплитуда и фаза колебаний уже установились. При вычислениях проводится «прямое» численное интегрирование уравнения движения электрона в заданном электромагнитном поле бегущей волны, поэтому программа может анализировать и нелинейные эффекты. Важно, что поле не задается только одной рабочей гармоникой, как это делается обычно. Для описания распределения продольной и поперечной составляющих напряженности ВЧ электрического поля бегущей волны в гребенке используется строгий аналитический метод конформных отображений [16-18]. В аналитических выражениях для поля учитываются сразу все гармоники и строго описываются особенности поля вблизи ребер металлических элементов гребенки (учет сингулярности).

Движение электронов предполагается трехмерным с учетом конечного фокусирующего магнитного поля. Можно также исследовать влияние начальных малых поперечных скоростей, полученных электронами при выходе из электронно-оптической системы электронной пушки. Наклон однородного фокусирующего поля по отношению к поверхности гребенки можно изменять и таким образом моделировать «клинотронный эффект» [1]. Движение электрона прослеживается до тех пор, пока электрон не попадет на металл гребенки или не выйдет из области, заданной для анализа.

Прослеживаются траектории электронов и изменение их скоростей в процессе движения. По изменению скорости электрона можно вычислить долю энергии, которую электрон отдает полю или отбирает у поля (работает закон сохранения энергии). Параметром является фаза ВЧ-поля в момент влета электрона (или компактного сгустка) в область ВЧ-поля.

Распределение амплитуды поля вдоль гребенки при расчете задается. Оно может задаваться любым и определяется из физических соображений. Например, при моделировании ламп бегущей волны поле можно задать экспоненциально нарастающим. При моделировании ЛОВ амплитуду поля по длине системы обычно описывают как убывающую по косинусу с нулем на коллекторном конце гребенки. После вычисления энергообмена по длине си- 
стемы распределение амплитуды поля по длине гребенки можно корректировать и приближаться к самосогласованному решению.

Покажем, что в отборнике мощности, работающем на отрицательной пространственной гармонике поля, при ожидаемых электронных КПД порядка единиц процента и малой длине отборника распределение амплитуды поля должно задаваться линейно убывающим по длине системы (от «пушечного» конца к «коллекторному»). Этот вывод не является неожиданным: именно такой вид имеет распределение амплитуды поля в обычных ЛОВ вблизи коллекторного конца замедляющей системы, где амплитуда ВЧ-поля мала.

Исследования модулирующей секции умножителя субмм-диапазона [7, 8] показали, что в узком слое пучка в компактный сгусток удается собрать около половины электронов слоя. Продольная длина сгустка на этом участке много меньше замедленной длины волны той гармоники модулятора, которая группирует электроны в сгустки. При анализе отборника на этом участке сгусток приближенно можно рассматривать как «точечный». Для этого его продольный размер должен быть в несколько раз меньше замедленной длины волны рабочей гармоники отборника $\left(\Lambda=\lambda \sqrt{U_{a}} / 505\right)$. При одинаковом ускоряющем напряжении для модулятора и отборника отношение длин их замедленных волн равно кратности умножения частоты. Для диапазона $\lambda=0,5$ мм мы предполагаем использовать 4-кратное умножение частоты (модулятор в 2-мм диапазоне волн).

Сгусток остается довольно компактным на участке длиной всего в несколько миллиметров. Поэтому отборник мощности в субммдиапазоне волн также должен иметь длину порядка нескольких миллиметров. Для диапазона $\lambda=0,5$ мм выберем длину отборника в 20 периодов гребенки (период равен 0,11 мм). При этом длина отборника составляет 2,2 мм, что соответствует размеру ожидаемой области компактности сгустка.

4. Распределение амплитуды поля по длине гребенки. Это распределение можно получить, приравняв изменение энергии за счет изменения амплитуды поля с изменением энергии электрона за счет изменения его скорости (закон сохранения энергии). Энергия поля $W$ пропорциональна квадрату амплитуды напряженности электрического поля $E(x)$ :

$W \sim[E(x)]^{2}, \frac{\partial W}{\partial x} \sim 2 E(x) \frac{\partial E(x)}{\partial x}$.

Предполагаем, что при движении вдоль гребенки электрон (сгусток) все время находится в тормозящей фазе поля гармоники и эта фаза мало изменяется за все время движения, поскольку скорость сгустка мало отличается от скорости гармоники. Тогда можем считать, что поле, действующее на сгусток, определяется только распределением поля по длине гребенки. Уравнение движения электрона в поле $E(x)$ :

$$
\begin{aligned}
& m \frac{\partial v(x)}{\partial t}=-e E(x), \\
& \frac{\partial v(x)}{\partial t}=\frac{\partial v(x)}{\partial x} \frac{\partial x}{\partial t} \approx \frac{\partial v(x)}{\partial x} v_{0},
\end{aligned}
$$

так как $v(x) \approx v_{0}$, где $v_{0}-$ скорость волны. Энергия электрона $W_{e}$ пропорциональна квадрату его скорости:

$$
\frac{\partial W_{e}}{\partial x} \sim 2 v(x) \frac{\partial v(x)}{\partial x} \approx 2 v_{0} \frac{\partial v(x)}{\partial x}=-2 \frac{e}{m} E(x) .
$$

Приравняем приращение энергии поля к потере энергии сгустком:

$\frac{\partial W}{\partial x} d x=\frac{\partial W_{e}}{\partial x} d x$

$E(x) \frac{\partial E(x)}{\partial x} d x=$ const $E(x) d x$.

Видно, что $E(x)$ сокращается. Для определения $E(x)$ получаем простое дифференциальное уравнение:

$$
\frac{\partial E(x)}{\partial x}=\text { const }, E(x)=\text { const }\left.x\right|_{L} ^{0} \text {. }
$$

Для $E(x)$ получилась линейная зависимость:

$E(x)=E(0)-[E(0)-E(L)] \frac{x}{L}$,

где $L$ - длина гребенки. В записи учтено, что электроны движутся навстречу волне и что в конце гребенки поле может быть отлично от нуля за счет обратной связи по ВЧ-полю.

Можно таким же способом показать, следующее: поскольку потери энергии в металле гребенки пропорциональны квадрату амплитуды поля, то их изменение по длине гребенки 
пропорционально амплитуде поля, как и в рассмотренных выше случаях. Поэтому учет потерь энергии не приведет к изменению линейной зависимости (5). Линейное распределение поля по длине гребенки в виде (5) будем задавать при численном исследовании.

5. Потери энергии в гребенке. Для моделирования движения электронов в отборнике мощности нужно задать амплитуду ВЧ-поля. Удобно задавать выходную мощность умножителя и соответствующую ей мощность потерь в резонаторах гребенки. По мощности потерь можно вычислить амплитуду поля в резонаторах и затем численно определить электронный КПД.

При расчете потерь энергии необходимо учитывать форму резонатора (рис. 2), сильно отличающуюся от обычной прямоугольной. Точно решить эту задачу сложно, и мы ограничимся лишь оценкой потерь.

В щелевом резонаторе возбуждается стоячая $T E M$-волна. Теоретические оценки [13], подтвержденные многочисленными экспериментами, показали, что дисперсионные характеристики гребенок с полукруглым дном щелей хорошо совпадают с расчетными полученными для гребенок со щелями прямоугольной формы, если считать глубиной полукруглого резонатора максимальное расстояние от поверхности гребенки до металла на дне щели (рис. 2). Поэтому можно считать, что распределение напряженности электрического поля стоячей $T E M$-волны на дне полукруглого резонатора будет таким же, как в прямоугольном резонаторе и $E$-поле обращается в нуль на дне щели на максимальном расстоянии от поверхности гребенки.

Распределение $E$ - и $H$-поля стоячей волны в щели запишем в виде:

$$
\begin{aligned}
& E_{x}=E_{\text {max }} \sin [k(y+h)], \\
& H_{z}=H_{\text {max }} \cos [k(y+h)] .
\end{aligned}
$$

Координата $y=0$ на поверхности гребенки и $y=-h$ на дне щелевого резонатора (рис. 2). Амплитуда $H$-поля в $T E M$-волне свя- зана с амплитудой $E$-поля соотношением [19]: $H_{\max }=E_{\max } / W_{0}$, где $W_{0}=120 \pi($ Ом $)$.

Распределение $H$-поля в щелевом резонаторе через среднее значение поля на «раскрыве» щели $E_{0}$ запишется так:

$$
H_{z}(y)=\frac{E_{0}}{W_{0} \sin (k h)} \cos [k(y+h)] .
$$

Постоянная распространения TEM-волны такая же, как в свободном пространстве: $k=2 \pi / \lambda$. Для вычисления мощности потерь энергии $P$ следует проинтегрировать возведенное в квадрат значение напряженности магнитного поля $H$ по поверхности щелевого резонатора:

$$
P=\frac{R^{s}}{2} \int_{s} H^{2} d s
$$

где $R^{s}$ - поверхностное сопротивление материала, из которого изготовлен резонатор. Для меди значение $R^{s}$ возьмем из [20], где $R^{s}-$ в Ом, а $\lambda$ - в см:

$R^{s} \approx \frac{0,047}{\sqrt{\lambda}}$.

Для учета шерховатости поверхности металла в мм-диапазоне в [20] рекомендуют увеличить $R^{s}$ для меди в 1,6 раза. Для диапазона $\lambda=0,5$ мм получим $R^{s} \approx 0,336$ Ом.

Мощность потерь энергии на поверхности полукруглого дна можно вычислить по формуле:

$$
\begin{aligned}
& P_{1}=\frac{R^{s}\left(E_{0}\right)^{2} B}{\left(W_{0}\right)^{2} \sin ^{2}(2 \pi h / \lambda)} \times \\
& \times \int_{0}^{\pi / 2} \cos ^{2}\left[\frac{2 \pi r}{\lambda}(1-\cos \varphi)\right] r d \varphi,
\end{aligned}
$$

где $r=l / 2 ; h$ - глубина щели; $l$ - ширина щели; $B$ - ширина гребенки по оси $z$. Интеграл в этой формуле следует определять численно; он равен $\pi r I_{1}$, где

$$
I_{1}=\frac{1}{2 N} \sum_{n=1}^{n=N} \cos ^{2}\left\{\pi \frac{l}{\lambda}\left[1-\cos \left(\frac{\pi}{2 N} n\right)\right]\right\} \text {. }
$$

Таблий 2

\begin{tabular}{|l|l|l|l|l|l|l|l|l|l|}
\hline$l / \lambda$ & 0,01 & 0,08 & 0,12 & 0,14 & 0,16 & 0,18 & 0,20 & 0,24 & 0,30 \\
$I_{1}$ & 0,4999 & 0,4929 & 0,4813 & 0,4788 & 0,4727 & 0,4659 & 0,4585 & 0,4422 & 0,4151 \\
\hline
\end{tabular}


Численные значения $I_{1}$ представлены в табл. 2 для $N=10000$.

Потери энергии в «линейной» части щели при заданном выше распределении $H_{z}(y)$, где $0>y>-(h-l / 2)$, определяются аналитически по формуле

$$
\begin{aligned}
& P_{2}=\frac{R^{s}\left(E_{0}\right)^{2} B}{\left(W_{0}\right)^{2} \sin ^{2}(2 \pi h / \lambda)} \times \\
& \times\left[\frac{\lambda}{8 \pi}\left(\sin \frac{4 \pi h}{\lambda}-\sin \frac{2 \pi l}{\lambda}\right)+\frac{h}{2}-\frac{l}{4}\right] .
\end{aligned}
$$

Мощность потерь энергии в одном резонаторе равна

$$
P_{N}=P_{1}+P_{2} \text {. }
$$

Если сравнить потери в исследуемом резонаторе с потерями в обычном щелевом резонатоpe c $h=\lambda / 4$, то при одинаковых $E_{0}$ они окажутся больше примерно в 2,8 раза.

Необходимо вычислить потери энергии во всех $N$ резонаторах гребенки, учитывая, что амплитуда $E$-поля линейно спадает по длине гребенки (5). Вычислив среднеквадратичное $E$-поле по длине гребенки, можем написать формулу для потерь энергии во всех $N$ резонаторах. Для этого достаточно в формулу для мощности потерь вместо $\left(E_{0}\right)^{2}$ подставить выражение

$$
N\left(E_{0}\right)^{2}\left[1-\frac{1-\gamma}{2}+\frac{(1-\gamma)^{2}}{3}\right] \text {, }
$$

где $\gamma=E(L) / E(0)-$ коэффициент обратной связи по ВЧ-полю. Видно, что при $\gamma=1$ (поле одинаково во всех резонаторах) потери просто увеличиваются в $N$ раз; при $\gamma=0$ (нет обратной связи) потери составляют примерно $83 \%$ от потерь при $\gamma=1$.

6. Определение безразмерной амплитуды поля для численного исследования. Безразмерную амплитуду поля, необходимую для численного исследования, свяжем с выходной мощностью умножителя. Задав выходную мощность, вычислим электронный КПД сгустка и затем определим ток электронного слоя, в котором происходит взаимодействие сгустков с полем, необходимый для получения заданной выходной мощности. Можно проводить оценку и другим способом: построить зависимость электронного КПД от выходной мощности и зависимость выходной мощности от имеюще- гося (заданного) тока сгустков при вычисленных значениях КПД. Пересечение этих двух зависимостей определит выходную мощность при заданном токе сгустков.

Безразмерная амплитуда $A$ связана со средней напряженностью электрического поля в щелевом резонаторе на уровне поверхности гребенки $E_{0}$ (поле на «раскрыве» щели) соотношением

$$
A=\frac{e}{m} \frac{E_{0}}{v_{0} \omega}
$$

где $e$ и $m$ - заряд и масса электрона; $v_{0}$ - начальная скорость электронов.

Генерируемая электронами мощность расходуется на мощность, выводимую из умножителя, и на компенсацию потерь энергии в резонаторах гребенки. Из-за отражений волны на концах гребенки система будет резонансной. Будем считать, что контурный КПД этого резонатора равен примерно $50 \%$. Определим, какой будет амплитуда поля $E_{0}$ при потерях в гребенке, равных выходной мощности умножителя $P$.

Следует особо отметить, что предполагаемое равенство потерь энергии в металле гребенке выходной мощности отборника определяет сильную обратную связь в системе по ВЧполю и, как следствие этого, большое увеличение амплитуды рабочей пространственной гармоники. Добротность такого резонатора в диапазоне волн 0,5 мм составляет около 160. Для того чтобы получить такой режим, требуется подобрать отражающие устройства на концах гребенки отборника, например, разместить на концах гребенки четвертьволновые отражающие щелевые резонаторы. Использование резонансной системы в отборнике мощности является эффективным средством для повышения электронного КПД отборника мощности умножителя в субмм-диапазоне волн. При этом, к сожалению, отборник будет эффективно работать только на дискретных резонансных частотах этого резонатора.

При вычислении приведенной амплитуды $E$-поля $A$ по формуле (9) удобно сразу выразить начальную скорость электронов через рабочее напряжение $U_{a}$ :

$$
A=\frac{e}{m} \frac{505 \lambda}{2 \pi c^{2} \sqrt{U_{a}}} E_{0},
$$



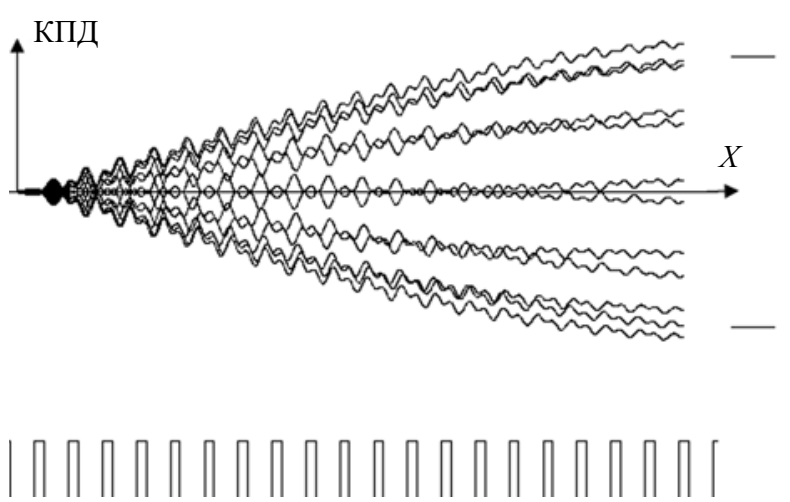

Puc. 3. Изменение скорости сгустков при их движении вдоль гребенки, пересчитанное в КПД сгустка (пропорционально изменению квадрата его скорости)

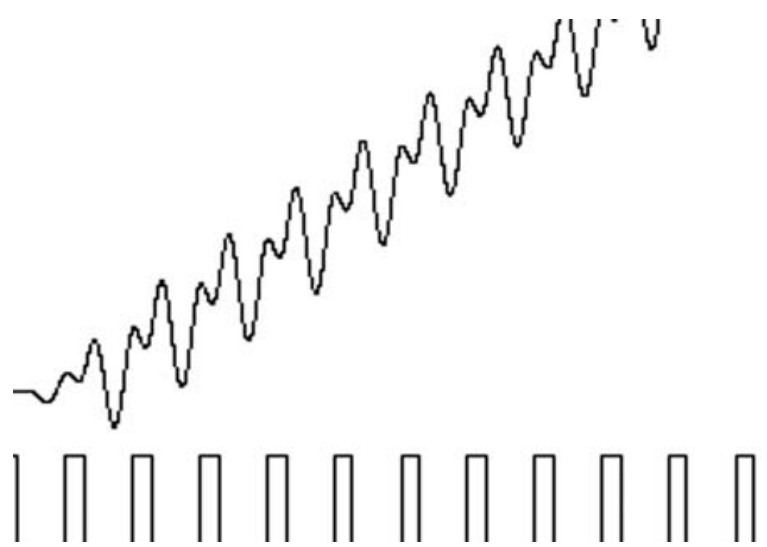

Puc. 4. Часть рис. 3 в увеличенном масштабе для одного сгустка

где $c$ - скорость света в вакууме. Амплитуду поля $E_{0}$ можно определить по заданной выходной мощности, равной мощности потерь энергии, из соотношений (6)-(8).

7. Вычисление электронного кПД для электронных сгустков. Программа «Клинотрон» определяет электронный КПД сгустка после прохождения им всей длины гребенки. Значение КПД пересчитывается по изменению скорости электрона на всем протяжении траектории. На рис. 3 показан пример работы программы «Клинотрон».

Амплитуда поля $E_{0}$ соответствует выходной мощности примерно 25 мВт; начальная скорость сгустков равна скорости -2-й пространственной гармоники волны. Показано изменение КПД сгустков при движении их вдоль гребенки, которая для масштаба условно показана внизу рисунка. Две горизонтальные метки справа соответствуют значению КПД $\pm 0,25 \%$. Параметром для серии зависимостей КПД от $x$ является фаза влета сгустка в поле гребенки. Положительные значения КПД означают передачу сгустком энергии ВЧ-полю; при отрицательном КПД сгусток забирает энергию у поля.

Для анализа важен только максимальный КПД, полученный при оптимальной фазе влета сгустка. Хотя ВЧ-поле считается заданным по амплитуде и фазе, на самом деле оно образуется в результате взаимодействия с анализируемым сгустком, и поэтому фаза поля всегда оптимальна для возбуждающего его сгустка. Ведь нельзя представить ситуацию, когда поле возбуждается сгустком, отбирающим энергию у поля, хотя такие случаи отражаются на рис. 3 при изменении фаз влета сгустков в заданное поле (отрицательные значения КПД). Изображение на графиках разных фаз влета сделано только для определения оптимальной фазы влета, значение которой заранее не известно.

Следует учитывать, что благодаря строгой кратности частот полей в модуляторе и отборнике все настоящие (а не расчетные) сгустки электронного потока влетают в поле отборника в одной и той же фазе, оптимальной для получения максимальной передаваемой полю мощности.

На рис. 3 видно, что сгусток, двигаясь в 7 раз медленнее основной гармоники бегущей волны, испытывает периодические возмущения при движении вдоль ламелей и щелей гребенки. Однако вследствие синхронизма сгустка со скоростью -2-й пространственной гармоники, в среднем он все время тормозится и отдает энергию ВЧ-полю, поскольку все время находится в тормозящей фазе рабочей гармоники. На рис. 4 показан фрагмент рис. 3 для одного сгустка в увеличенном масштабе.

Проведем анализ работы отборника на конкретном примере с набором параметров, который можно реализовать экспериментально.

Набор параметров следующий: рабочая длина волны $\lambda=0,5$ мм; рабочее напряжение при работе на -2-й пространственной гармонике $U_{a}=4000 \mathrm{~B}$; фазовый сдвиг основной гармоники на период гребенки $\varphi=0,5 \pi$; индукция фокусирующего магнитного поля 1 Тл (соотношение рабочей и циклотронной частоты составляет около 20); период гребенки с полукруглым дном щелей $S=0,11$ мм; ширина щели $l=0,08$ мм; глубина щели $h=0,06$ мм; число 
резонаторов гребенки $N=20$; ширина гребенки 2,5 мм. В начале гребенки поле нарастает от нуля до значения $E_{0}$ не скачком, а на протяжении двух периодов гребенки; начальная поперечная скорость сгустка равна нулю; обратная связь по ВЧ-полю в отборнике 30 \% (задана амплитуда поля в конце гребенки); амплитуда $E$-поля линейно уменьшается по длине гребенки от «пушечного» конца к «коллекторному» концу; мощность потерь энергии в гребенке равна выходной мощности умножителя; фаза влета сгустка в ВЧ-поле задается для 12 значений за период ВЧ-поля отборника (равномерно).

Для численного счета необходимо задать приведенную амплитуду ВЧ-поля $A$ согласно выражению (10). В эту формулу входит величина $E_{0}$, которую следует определить по формуле потерь в гребенке, считая, что мощность потерь равна выходной мощности умножителя. Выходную мощность умножителя зададим как параметр и определим, каким будет электронный КПД сгустка при напряженности ВЧ-поля в гребенке, соответствующей заданной выходной мощности. Результаты численного анализа КПД по программе «Клинотрон» для разной выходной мощности при оптимальной фазе влета представим в табл. 3 .

Приведем формулу для определения напряженности поля $E_{0}$ на первом резонаторе гребенки (в вольтах на метр) через выходную мощность умножителя $P$ (в ваттах), с учетом всех полученных выше формул:

$$
\begin{aligned}
& \left(E_{0}\right)^{2}=\frac{P}{N\left[1-\frac{1-\gamma}{2}+\frac{(1-\gamma)^{2}}{3}\right]} \times \\
& \times \frac{\text { const }}{\left[\frac{\lambda}{8 \pi}\left(\sin \frac{4 \pi h}{\lambda}-\sin \frac{2 \pi l}{\lambda}\right)+\frac{h}{2}-\frac{l}{4}+\frac{\pi l}{2} I_{1}\right] B},
\end{aligned}
$$

где $\gamma=E_{0}(L) / E_{0}(0)$ - коэффициент обратной связи по ВЧ-полю; $I_{1}-$ значение интеграла из табл. 2 ; const $=\frac{\left(W_{0}\right)^{2} \sin ^{2}(2 \pi h / \lambda)}{R^{s}} ; h-$ глубина щелевого резонатора; $l$ - ширина щели этого резонатора; $B$ - ширина гребенки; $N$ - число резонаторов гребенки; $\lambda$ - длина волны; $R^{s}$ поверхностное сопротивление (для $\lambda=0,5 \mathrm{мm}$ $R^{s}=0,336$ Ом); $W_{0}=120 \pi$ Ом. Все линейные размеры - в метрах.

Подставив приведенный выше набор параметров в эту формулу, получим

$\left(E_{0}\right)^{2}=0,6164 \cdot 10^{8} P$,

где $P$ измеряется в милливаттах, а $E_{0}-$ в вольтах на метр.

Подставив значение параметров в формулу (10), получим выражение для безразмерной приведенной амплитуды $A$ в удобном для вычислений виде:

$A=1,242 \times 10^{-9} E_{0}$,

где $E_{0}$ выражено в вольтах на метр.

Результаты численного исследования зависимости электронного КПД сгустков от выходной мощности умножителя (от амплитуды поля в гребенке, соответствующей этой мощности) представлены в табл. 3.

Значение КПД 1 вычислено для случая, когда сгустки входят в пространство над гребенкой на расстоянии 0,01 мм от ее поверхности и в дальнейшем движутся параллельно этой поверхности. Значение КПД 2 вычислено для случая, когда используется клинотронный эффект - фокусирующее магнитное поле слегка наклонено относительно поверхности гребенки так, чтобы сгустки приближались к поверхности гребенки и даже оседали на ней. Сгустки входят в ВЧ-поле на расстоянии 0,005 мм от

Таблица 3

\begin{tabular}{|l|c|c|c|c|c|c|c|}
\hline$P, \mathrm{мBт}$ & 1 & 5 & 10 & 25 & 50 & 75 & 100 \\
$\left(E_{0}\right)^{2} \times 10^{-8}$ & 0,6164 & 3,082 & 6,164 & 15,41 & 30,82 & 46,23 & 61,64 \\
$E_{0} \times 10^{-4} \mathrm{~B} / \mathrm{M}$ & 0,7851 & 1,7556 & 2,4827 & 3,9256 & 5,5516 & 6,7993 & 7,8511 \\
$A \times 10^{5}$ & 0,9751 & 2,1804 & 3,0835 & 4,8756 & 6,8951 & 8,4447 & 9,7511 \\
КПД $1, \%$ & 0,051 & 0,12 & 0,16 & 0,24 & 0,35 & 0,50 & 0,55 \\
КПД $2, \%$ & 0,105 & 0,24 & 0,34 & 0,53 & 0,76 & 0,94 & 1,00 \\
$P$ от $J_{1}, \mathrm{мBт}$ & 2,55 & 6 & 8 & 12 & 17,5 & 25 & 27,5 \\
$P$ от $J_{2}, \mathrm{мBт}$ & 5,1 & 12 & 16 & 24 & 35 & 50 & 55 \\
\hline
\end{tabular}


поверхности гребенки и оседают на гребенке в области последнего 20-го резонатора. Заметно значительное повышение КПД. В двух нижних строках указана мощность, которую можно получить от сгустков при вычисленном КПД для двух значений сгруппированного тока пучка $J_{1}$ и $J_{2}$. Значение $J_{1}$ соответствует плотности тока с катода $20 \mathrm{~A} / \mathrm{cm}^{2}$, а $J_{2}$ - плотности тока $40 \mathrm{~A} / \mathrm{cm}^{2}$.

Сгруппированный ток рассчитывался следующим образом. Предполагается, что будет использоваться катод размером $0,1 \times 2,5 \mathrm{mм}^{2}$. В группирователе и отборнике эффективно используется только слой электронов толщиной около 0,01 мм. Ранее при исследовании модулятора было установлено, что в плотные сгустки удается собрать около половины электронов этого тонкого слоя [7]. Следовательно, при плотности тока с катода $20 \mathrm{~A} / \mathrm{cm}^{2}$ сгруппированный ток составит примерно 2,5 мА. При напряжении 4000 В мощность сгруппированного тока равна 10 Вт. Умножив эту величину на КПД, получим мощность, генерируемую электронным пучком. Следует учитывать, что только половина этой мощности используется на поддержание ВЧ-поля гребенки, а половина уходит в вывод энергии. Поэтому величину мощности, создающей ВЧ-поле, следует уменьшить еще в 2 раза. Мощность, отдаваемая электронами полю, может рассчитываться по формуле

$P=5 \times($ КПД),

где $P$ измеряется в ваттах.

При плотности тока с катода $40 \mathrm{~A} / \mathrm{cm}^{2}$ эта мощность удвоится. В двух последних строках таблицы приведена мощность для КПД электронов, при параллельном поверхности гребенки пучке (без клинотронного эффекта).

Данные табл. 3 позволяют определить, какой режим возможен при заданном токе. Очевидно, что мощность, отдаваемая электронами ВЧполю при вычисленном КПД, должна быть не меньше, чем та, которую предполагалось расходовать на поддержание поля при вычислении КПД. Сравнение данных верхней строки (предполагаемая мощность) и двух нижних строк (полученная мощность) показывает, что при $J_{1}$ «баланс» энергии соответствует выходной мощности умножителя, превышающей 5 мВт, но не более 10 мВт, и для плотности тока $J_{2}$ при мощности умножителя примерно $25 \mathrm{mBT}$.

Данные с учетом клинотронного эффекта (когда сгустки оседают на гребенку в области последнего резонатора) для тех же плотностей тока приведены в табл. 4 .

Сравнение данных верхней строки и двух нижних строк показывает, что равенство расходуемой и поступающей мощности достигается при выходной мощности умножителя около 25 мВт при плотности тока с катода $20 \mathrm{~A} / \mathrm{cm}^{2}$ и около 100 мВт при плотности тока с катода $40 \mathrm{~A} / \mathrm{cm}^{2}$.

В настоящее время имеются катоды с плотностью тока до $50 \mathrm{~A} / \mathrm{cm}^{2}$, однако с повышением плотности тока снижается срок службы катода.

8. О формировании электронных сгустков. Оценка выходной мощности умножителя проводилась с учетом реальных параметров, однако при расчетах предполагалось, что уже имеется очень тонкий слой электронов, сформированных в достаточно компактные сгустки. Для получения таких пучков требуется оптимизация параметров группирователя и специальные методы формирования ленточного пучка.

Хорошие результаты может дать отсечение половины пучка вблизи его плоскости симметрии на охлаждаемом промежуточном коллекторе-коллиматоре перед проходом пучка в эквипотенциальную область отборника мощности. В плоскости симметрии пучка отсутствуют пульсации, которые электроны получают при выходе из катода и после прохождения анодного отверстия электронной пушки (при условии, что катод расположен симметрично относительно анодного отверстия). Поэтому в уз-

\section{Таблий 4}

\begin{tabular}{|l|c|c|c|c|c|c|c|}
\hline$P$, мВт & 1 & 5 & 10 & 25 & 50 & 75 & 100 \\
$A \times 10^{5}$ & 0,9751 & 2,1804 & 3,0835 & 4,8756 & 6,8951 & 8,4447 & 9,7511 \\
КПД $2, \%$ & 0,105 & 0,24 & 0,34 & 0,53 & 0,76 & 0,94 & 1,00 \\
$P$ от $J_{1}$, мВт & 5,25 & 12 & 17 & 26,5 & 38 & 47 & 50 \\
$P$ от $J_{2}$, мВт & 10,5 & 24 & 34 & 53 & 76 & 94 & 100 \\
\hline
\end{tabular}


ком слое обрезанной части пучка у поверхности гребенки пульсаций также не должно быть. Оценки остаточных пульсаций в этом слое за счет пространственного заряда оставшейся половины пучка показывают, что при индукции фокусирующего магнитного поля в 1 Тл пульсации границ меньше 0,001 мм. Обычно используются электронные пушки с минимальной толщиной пучка около 0,1 мм. Для работы субмиллиметрового отборника мощности используется не весь ленточный пучок, а только его десятая часть толщиной $0,01 \ldots 0,015$ мм.

Следует отметить, что похожий прием улучшения качества пучка использовался ранее при создании клинотронов субмм-диапазона волн [1]. Перед гребенкой генератора устанавливался так называемый «вкладыш», который при наклоне пучка фокусирующим магнитным полем мог принимать на себя часть ленточного пучка с большими пульсациями и «срезать» ее. Правда, там не предполагалось обрезать пучок по плоскости симметрии и «вкладыш» только защищал первые ламели гребенки. Возможно, именно этим конструктивным решением объясняется достижение на таком клинотроне рекордно короткой длины волны $\lambda \approx 0,55$ мм.

Проведенное исследование следует рассматривать как оценку верхнего предела достижимых КПД и выходной мощности умножителя. Необходимо учитывать неизбежное «расплывание» сформированных сгустков в продольном и поперечном направлениях при большой плотности пространственного заряда в сгустке. В наших оценках сгустки предполагались достаточно компактными (много меньше замедленной длины волны рабочей гармоники).

9. Оценка перехода в диапазон $\lambda=0,3$ мм. Для оценок перехода в диапазон $\lambda=0,3$ мм следует учесть увеличение потерь энергии в металле, пропорциональное квадратному корню из частоты. Поскольку в разд. 3 были определены примерные размеры гребенки для этого диапазона масштабным моделированием из диапазона $\lambda=0,5$ мм, то в формулах для анализа движения сгустков в гребенке с измененными размерами поменяется только величина поверхностного сопротивления $R^{S}$. Оно увеличится пропорционально квадратному корню из отношения длин волн - в 1,26 раза. Во столько же раз уменьшится квадрат амплитуды на- пряженности $E$-поля $E_{0}$. Само поле изменится как корень из этой величины - в 1,12 раза. Приведенная амплитуда $A$, необходимая для численного интегрирования уравнений движения (пропорциональная $E_{0}$ ), также уменьшится только на $12 \%$ по сравнению со случаем $\lambda=0,5$ мм. Результаты такого изменения можно видеть в табл. 2-4 (при изменении $A$ ).

Таким образом, при масштабном моделировании размеров и режима отборника результаты расчетов КПД количественно изменятся незначительно по сравнению с диапазоном $\lambda=0,5$ мм. Трудности возрастут при формировании электронного потока и компактных сгустков при уменьшении всех размеров в 1,66 раза. Основная трудность - ввести модулированный электронный поток в область ВЧполя рабочей гармоники, ширина которой, по оценкам, приведенным в начале статьи, составляет менее 0,01 мм. Это же замечание относится и к диапазону $\lambda=0,5$ мм.

Выводы. Основной целью проведенных в работе исследований является оценка возможности создания электровакуумных умножителей частоты в коротковолновой части субммдиапазона волн и определение параметров таких умножителей при использовании существующих экспериментальных возможностей. Для исследования отборника мощности субммдиапазона был применен достаточно надежный численный метод, специально разработанный для таких задач. Важным преимуществом применяемого метода является большая доля использования аналитических результатов, в частности, при вычислении распределения полей в замедляющей системе. Благодаря этому обстоятельству время вычисления одного варианта сочетания параметров составляет десятки секунд, а не часы, как при использовании прямых численных методов в сложных универсальных программах. Для повышения надежности результатов в работе большое внимание уделяется максимально точному вычислению входных параметров задачи, в частности, вычислению потерь энергии в гребенках с малой глубиной щелевых резонаторов с полукруглым дном щелей.

Следует отметить, что для повышения КПД электронов в отборнике предполагалось использовать резонансную замедляющую систему с 
сильной обратной связью по ВЧ-полю (потери энергии в гребенке равны мощности, выводимой из отборника). При этом существенно возрастает амплитуда рабочей гармоники.

Детальные исследования позволили подобрать режим отборника и параметры замедляющей системы, допускающие реализацию при существующих технологических возможностях. Это является важным для освоения диапазона $\lambda<0,55$ мм, поскольку сейчас в этом диапазоне в Украине практически не производятся приборы с заметной выходной мощностью.

Результаты наших исследований позволяют сделать вывод, что возможно создание отборника мощности при вполне выполнимых требованиях к технологическим возможностям, с выходной мощностью в десятки милливатт в непрерывном режиме в диапазоне $0,3 \leq \lambda \leq$ $\leq 0,5$ мм. В настоящее время такие параметры могут считаться хорошими.

Однако для реализации таких возможностей отборника мощности требуется обеспечить группировку компактных сгустков электронов в тонком слое пучка толщиной 0,01 мм. Это очень сложная задача, которая, кроме исследо- вания группирователя сгустков и оптимизации его режима, требует новых решений по формированию тонких электронных слоев с минимальными пульсациями границ. Для выполнения жестких требований к качеству фокусировки пучков может потребоваться использование новых необычных методов их формирования. Один из таких методов схематически описан в разд. 8. Предлагается использовать промежуточный охлаждаемый коллектор электронов перед входом пучка в пространство отборника мощности. Срезая с помощью этого коллектораколлиматора половину пучка на уровне его плоскости симметрии (где, в принципе, отсутствуют пульсации в движении электронов), можно радикально решить задачу однородности тонкого электронного слоя, прилегающего к поверхности гребенки отборника.

Кроме новых конструктивных решений и оптимизации параметров группирователя и отборника мощности, при изготовлении электровакуумных умножителей субмм-диапазона волн требуется значительно повысить точность изготовления деталей и точность сборки приборов.

\section{БИБЛИОГРАФИЧЕСКИЙ СПИСОК}

1. Левин Г.Я., Бородкин А.И., Кириченко А.Я., Усиков А.Я., Чурилова С.А. Клинотрон. Киев: Наук. думка, 1992.200 с.

2. Dayton J.A.Ir., Mearini G.T., Kory C.L. Diamond Based Sub Millimeter Back Wave Oscillator. IEEE Int. Vacuum Electronics Conf. (IVEC 2004): conf. dig. (Monterey, CA, USA, 27-29 April 2004) Monterey, 2004. P. 71-72.

3. Лампы обратной волны миллиметрового и субмиллиметрового диапазонов волн. Под ред. Н.Д. Девяткова. Москва: Радио и связь, 1985. 135 с.

4. Ives L., Kory C., Read M. Development of Terahertz Backward Wave Oscillators. IEEE Int. Vacuum Electronics Conf. (IVEC 2004): conf. dig. (Monterey, CA, USA, 27-29 April 2004) Monterey, 2004. P. 68-69.

5. Еремка В.Д. Умножители частоты с наклонным электронным потоком. Радиофизика и электрон.: сб. науч. тр. Ин-т радиофизики и электрон. НАН Украины. Харьков: 2007. Т. 12, спец. вып. С. 81-103.

6. Еремка В.Д., Кириченко А.Я. Экспериментальное исследование миллиметрового умножителя частоты с высоким коэффициентом умножения. Тр. ИРЭ АН УССР. Харьков: 1970. Т. 18. С. 138-148.

7. Мильчо М.В. Группировка электронов в генераторе типа «клинотрон». Клинотрон как умножитель частоты. Радиофизика и электроника. 2015. Т. 6(20), № 2. С. 54-60. DOI: https://doi.org/10.15407/rej2015.02.054.

8. Мильчо М.В., Тищенко А.С., Завертанный В.В., Лопатин И.В., Терехин С.Н. Клинотрон - умножитель частоты в субмиллиметровом диапазоне длин волн ( $\lambda=0,93$ мм). Радиофизика и электроника. 2015. Т. 6(20), № 2. С. 61-67.

9. Альтшулер Ю.Г., Татаренко А.С. Лампы малой мощности с обратной волной. Москва: Сов. радио, 1963. 296 с.

10. Мильчо М.В., Ефимов Б.П., Завертанный В.В., Гончаров В.В. Особенности режимов работы генераторов типа клинотрон. Радиофизика и электроника: сб. науч. тр. Ин-т радиофизики и электрон. НАН Украины. Харьков: 2005. T. 10, № 3. C. 435-440.

11. Кириленко А.А., Сенкевич С.Л., Стешенко С.А. Анализ трехмерных замедляющих систем на основе метода обобщенных матриц рассеяния. Радиофизика и электроника: сб. науч. тр. Ин-т радиофизики и электрон. НАН Украины. Харьков: 2007. Т. 12, спец. вып. С. 122-129.

12. Hongzhu Xi, Zhaochang He, Jianguo Wang, Rong Li, Gang Zhu, Zaigao Chen, Jinsong Liu, Luwei Liu, Hao Wang. A continuous - wave clinotron at $0.26 \mathrm{THz}$ with sheet electron beam. Physics of plasmas. 2017. Vol. 24, Iss. 3. P. 033105(6 p.).

13. Мильчо М.В. О слабом влиянии скруглений углов в резонаторах гребенки на ее дисперсионные характеристики. Радиофизика и электроника: сб. науч. тр. Ин-т радиофизики и электрон. НАН Украины. Харьков: 2006. Т. 11, № 2. С. $190-195$.

14. Мильчо М.В. Учет трехмерного движения электронов в генераторах типа «клинотрон». Ч. 1. Методика численного исследования. Радиофизика и электроника. Харьков: 2010. Т. 1(15), № 3. С. 83-91. 
15. Мильчо М.В. Учет трехмерного движения электронов в генераторах типа «клинотрон». Ч. 2. Анализ реальных приборов. Радиофизика и электроника. Харьков: 2010. Т. 1(15), № 4. С. 58-65.

16. Мильчо М.В. Метод конформных отображений для расчета высокочастотных электромагнитных полей в замедляющих системах. Ч. 1. Случай больших замедлений. Радиофизика и электроника: сб. науч. тр. Ин-т радиофизики и электрон. НАН Украины. Харьков: 2003. Т. 8, № 1. С. 136-147.

17. Мильчо М.В. Метод конформных отображений для расчета высокочастотных электромагнитных полей в замедляющих системах. Ч. 2. Электродинамические решения эквивалентные электростатическим. Радиофизика и электрони$\kappa a:$ сб. науч. тр. Ин-т радиофизики и электрон. НАН Украины. Харьков: 2003. Т. 8, № 2. С. 259-268.

18. Мильчо М.В. Метод конформных отображений для расчета высокочастотных электромагнитных полей в замедляющих системах. Ч. 3. Анализ конкретных систем. Радиофизика и электроника: сб. науч. тр. Ин-т радиофизики и электрон. НАН Украины. Харьков: 2003. Т. 8, № 3. С. 374-385.

19. Никольский В.В. Теория электромагнитного поля. Москва: Высшая школа, 1961. 372 с.

20. Ширман Я.Д. Радиоволноводы и объемные резонаторы. Москва: Связьиздат, 1959. 379 с.

Стаття надійшла 08.01.2019

\section{REFERENCES}

1. Levin, G.Y., Borodkin, A.I., Kirichenko, A.Y., Usikov, A.Y. ed., Churilova, S.A., 1992. Klynotron. Kiev: Naukova Dumka Publ. (in Russian).

2. Dayton, J.A.Ir., Mearini, G.T., Kory, C.L., 2004 Diamond Based Sub Millimeter Back Wave Oscillator. In: IEEE Int. Vacuum Electronics Conf. (IVEC 2004): conf. dig. Monterey, CA, USA, 27-29 April 2004, pp. 71-72.

3. Deviatkov N.D. ed., 1985. BWT of sub- $m m$ wave-band. Moscow: Radio i Sviaz' Publ. (in Russian).

4. Ives, L., Kory, C., Read, M., 2004. Development of Terahertz Backward Wave Oscillators. In: IEEE Int. Vacuum Electronics Conf. (IVEC 2004): conf. dig. Monterey, CA, USA, 27-29 April 2004, pp. 68-69.

5. Yeryomka, V.D., 2007. Inclined-electron flow frequency multiplier. In: V.M. Yakovenko, ed. 2007. Radiofizika i elektronika. Kharkov: IRE NAS of Ukraine Publ. 12(spec. iss.), pp. 81-103 (in Russia).

6. Yeryomka, V.D., Kirichenko, A.Y., 1970. Experimental research of a millimeter waveband frequency multiplier of a high multiplication factor. Proc. of IRE AS UkrSSR, Kharkov. 18, pp. 138-148 (in Russian).

7. Mil'cho, M.V., 2015. Electron bunching in the clinotron type oscillators. "Clinotron" as a frequency multiplier. Radiofiz. elektron., 6(20(2), pp. 54-60 (in Russian). DOI: https://doi.org/10.15407/rej2015.02.054.

8. Mil'cho, M.V., Tischenko, A.S., Zavertaniy, V.V., Lopatin, I.V., Teriohin, S.N., 2015. Clinotron as a frequency multiplier in the sub-mm wave-bend ( $\lambda=0,93 \mathrm{~mm})$. Radiofiz. elektron., 6(20(2), pp. 61-67 (in Russian).

9. Altshuler, Yu.G., Tatarenko, A.S., 1963. Backward wave tubes with low power. Moscow: Sovetskoe Radio Publ. (in Russian).

10. Mil'cho, M.B., Efimov, B.P., Zavertanniy, V.V., Goncharov, V.V., 2005. Peculiar properties of operating modes of klinotron-type oscillators. In: V.M. Yakovenko, ed. 2005. Radiofizika i elektronika. Kharkov: IRE NAS of Ukraine Publ. 10(3), pp. 435-440 (in Russian).

11. Kirilenko, A.A., Senkevich, S.L., Steshenko, S.O., 2007. Dispersion analysis of three dimensional slow-wave structures on basis of generalized scattering matrix method. In: V.M. Yakovenko, ed. 2007. Radiofizika i elektronika. Kharkov: IRE NAS of Ukraine Publ. 12(spec. iss.), pp. 122-129 (in Russian).

12. Hongzhu, Xi, Zhaochang, He, Jianguo, Wang, Rong, Li, Gang, Zhu, Zaigao, Chen, Jinsong,Liu, Luwei, Liu, Hao, Wang, 2017. A continuous - wave clinotron at $0.26 \mathrm{THz}$ with sheet electron beam. Phys. Plasma, 24(3), pp. 033105(6 p.).

13. Mil'cho, M.V., 2006. On the weak effect of the rounding of corners in comb resonators on the comb dispersion characteristics. In: V.M. Yakovenko, ed. 2007. Radiofizika i elektronika. Kharkov: IRE NAS of Ukraine Publ. 11(2), pp. 190-195 (in Russian).

14. Mil'cho, M.V., 2010. 3D motion of electrons in klynotron oscillator. Ch. 1. Numerical method analysis. Radiofizika i elektronika, 1(15)(3), pp. 83-91 (in Russian).

15. Mil'cho, M.V., 2010. 3D motion of electrons in klynotron oscillator. Ch. 2. Real oscillator analyses. Radiofizika i elektronika, 1(15)(4), pp. 58-65 (in Russian).

16. Mil'cho, M.V., 2003. The conformal mapping method for analysis high-frequency electro-magnetic fields in slow-wave structures. Ch. 1. The case of large slow-downs. In: V.M. Yakovenko, ed. 2003. Radiofizika i elektronika. Kharkov: IRE NAS of Ukraine Publ. 8(1), pp. 136-147 (in Russian).

17. Mil'cho, M.V., 2003. The conformal mapping method for analysis high-frequency electro-magnetic fields in slow-wave structures. Ch. 2. Electro-dynamics solutions being equivalent to electrostatic ones. In: V.M. Yakovenko, ed. 2003. Radiofizika i elektronika. Kharkov: IRE NAS of Ukraine Publ. 8(2), pp. 259-268 (in Russian).

18. Mil'cho, M.V., 2003. The conformal mapping method for analysis high-frequency electro-magnetic fields in slow-wave structures. Ch. 3. The concrete slow-wave systems analysis. In: V.M. Yakovenko, ed. 2007. Radiofizika i elektronika. Kharkov: IRE NAS of Ukraine Publ. 8(3), pp. 374-385 (in Russian).

19. Nikol'skiy, V.V., 1961. Electromagnetic field theory. Moscow: Visshaya Shkola Publ. (in Russian).

20. Shirman, Y.D., 1959. Radio waveguides and cavity resonators. Moscow: Svyaz'izdat Publ. (in Russian).

Received 08.01.2019 
M.V. Mil'cho

O.Ya. Usikov Institute for Radiophysics and Electronics of NAS of Ukraine

12, Acad. Proskury st., Kharkov, 61085, Ukraine

RESEARCH AND DESIGN OF POWER CATCHER

FOR ELECTRO-VACUUM FREQUENCY MULTIPLIER

OF SUB-MILLIMETER WAVE RANGE

Subject and purpose. Presently, there is a problem of creation of simple in exploitation generators for a sub-millimeter range, especially in its short-wave part. Electro-vacuum generators of the type BWT and klynotron work for long-wave part of sub-millimeter range. Difficulties of design are increasing with shortening of wave length. The sizes of the slow-wave structures diminish, requirements to the density of current of electronic bunches and to quality of their focusing increase. The use of electro-vacuum multipliers of frequency can weaken these requirements considerably. The paper describes a numerical study of the catcher for the electro-vacuum frequency multipliers of sub-millimeter wave range. The aim of the research is to estimate the achievable output power of frequency multipliers and search for optimal parameters for their realization in sub-millimeter wave range.

Methods and methodology. For calculation of the catcher efficiency, the analysis of three-dimension motion of electronic bunch in set field of the slowing wave system is conducted. For the numerical integration of the electron motion equation, the universal program "Klynotron" is used. This program was developed by us before for BWT and klynotron research. In this program, many parameters of the real task are taken into account. For description of spatial distribution longitudinal and transversal constituents of electric field tension, the exact analytical method of conformal mapping is used. Moreover, all spatial harmonics of the field are taken into account in analytical expressions at once and the field singularity is exactly described nearby the ribs of metallic elements.

Results. A numerical study of the influence of the catcher parameters on the output parameters of the multiplier was carried out. The mode and design parameters of the catcher of the frequency multiplier for the wave ranges $\lambda=0.5 \mathrm{~mm}$ and $\lambda=0.3 \mathrm{~mm}$ are determined.

Conclusion. The study showed that with the existing technological capabilities it is realistic to create frequency multipliers for wave ranges $\lambda=0.5 \ldots 0.3 \mathrm{~mm}$ with an output power of the order of tens of milliwatts. A necessary condition for creation of such devices is optimization of parameters of buncher of electronic clots in the frequency multiplier

Key words: electronics, klynotron, bunching, frequency multiplier, numerical calculation.

\section{М.В. Мільчо}

Інститут радіофізики та електроніки ім. О.Я. Усикова НАН України

12, вул. Акад. Проскури, Харків, 61085, Україна

\section{ДОСЛІДЖЕННЯ ТА ПРОЕКТУВАННЯ ВІДБІРНИКА \\ ПОТУЖНОСТІ ЕЛЕКТРОВАКУУМНОГО ПОМНОЖУВАЧА \\ ЧАСТОТИ СУБМІЛІМЕТРОВОГО ДІАПАЗОНУ}

Предмет і мета роботи. Зараз існує проблема створення зручних для експлуатації генераторів для освоєння короткохвильової частини субміліметрового діапазону хвиль. У генераторах типу ЛЗХ (лампа зворотної хвилі) та клинотронах при скороченні довжини хвиль зростають труднощі, що пов'язані із зменшенням розмірів систем сповільнення хвиль та зростанням вимог до якості електронних потоків. Ці вимоги можливо значно послабити при використанні помножувачів частоти. У статті описано числове дослідження роботи відбірника потужності у електровакуумних помножувачах частоти субміліметрового діапазону хвиль. Мета цих досліджень - оцінка вихідної потужності, яку можливо досягти у таких помножувачах частоти.

Методи та методологія роботи. Для обчислення ККД відбірника потужності проведено аналіз тривимірного руху електронних ущільнень у заданому електромагнітному полі хвилі, що біжить у системі для сповільнення хвиль типу «гребінка». Для числового аналізу використано програму «Клинотрон», яку ми розробили раніше. Для опису розподілу у просторі напруженості електричного поля використано строгий аналітичний метод конформних відображень. При цьому в аналітичних виразах враховано одразу всі просторові гармоніки поля і строго описано сингулярність поля поблизу ребер гребінки.

Результати роботи. Проведено числове дослідження впливу параметрів відбірника потужності на вихідні параметри помножувача. Визначені режим та конструктивні параметри відбірника потужності помножувачів частоти для діапазонів хвиль $\lambda=0,5$ мм и $\lambda=0,3$ мм.

Висновок. Проведене дослідження показало, що при наших технологічних можливостях цілком реально створити помножувачі частоти в діапазонах хвиль $\lambda=0,5 \ldots 0,3$ мм з вихідною потужністю порядку десятків міліват. Необхідна також оптимізація модулятора та секції для групування електронних ущільнень у помножувачі частоти.

Ключові слова: електроніка, клинотрон, групування, помножувач частоти, числовий розрахунок. 